durch Bildung anomaler Mischkristalle der Silberhalogenide mit Blei- und Cadmiumhalogeniden Leerstellen im Kationengitter in bekannter Zahl herzustellen und damit die Größen $E_{0}$ und $U$ in (2) getrennt zu ermitteln. Es lag nahe, diese Ergebnisse durch Messungen der Druckabhängigkeit der Leitfähigkeiten in anomalen Mischkristallen zu prüfen. Wenn die Zahl der Leerstellen durch die Konzentration des Zusatzes fest vorgegeben ist, kann die Temperaturabhängigkeit der Leitfähigkeit nur noch durch $U$ gegeben sein, wie aus den Messungen von Koch und Wagner zu ersehen ist. Dann mul auch der Druckeffekt durch die Änderung von $U$ allein gegeben sein und entsprechend geringer ausfallen als im reinen Silberhalogenid.

Messungen dieses Effektes, die bereits vor 10 Jahren begonnen worden waren, konnten nun zum Abschluf gebracht werden. Dabei ergab sich beim.Silberchlorid, wie zu erwarten, eine kleinere Druckabhängigkeit im Falle anomaler Mischkristalle mit Bleichlorid als mit reinem Silberchlorid.

Im einzelnen wurde gefunden:

$$
\begin{array}{cc}
\mathrm{AgCl}: t=256-313^{\circ} \mathrm{C} & -\frac{1}{\sigma} \frac{\partial \sigma}{\partial p}=3,0 \cdot 10^{-4}, \\
\mathrm{AgCl}+0,42 \mathrm{Mol}-\% \mathrm{PbCl}_{2}: & -\frac{1}{\circ} \frac{\partial \sigma}{\partial p}=1,3 \cdot 10^{-4} . \\
t=293^{\circ} \mathrm{C} & -
\end{array}
$$

J ost und Nehlep ${ }^{12}$ hatten im Mittel gefunden: $\mathrm{AgCl}$ bei $300^{\circ} \mathrm{C} \quad-\frac{1}{\sigma} \frac{\partial \sigma}{\partial p}=2,5 \cdot 10^{-4}$.

Der Druckeffekt der anomalen Mischkristalle beträgt also im Mittel nur etwa $40 \%$ desjenigen des reinen Salzes.

Stellt man $E$ bzw. $U$ (vgl. die theoretischen Rechnungen von $\mathrm{Jost}^{3}, \mathrm{Schottky^{4 }}$, Mott-Littleto $\mathrm{n}^{5}$ ) mittels einer zweikonstantigen Formel dar:

$$
\begin{aligned}
& U=\frac{A}{r}+\frac{B}{r^{n}}, \\
& U=\frac{A}{r}+B \exp (g / r),
\end{aligned}
$$

wobei $n$ bzw. @ aus anderweitigen Beziehungen entnommen sind, so erlaubt die Kombination der KochWagnerschen Resultate mit der Bestimmung ron $-\frac{1}{\sigma} \frac{\partial \sigma}{\partial p}$, welche auf eine Bestimmung von $\partial E / \partial r$ bzw. $\partial U / \partial r$ herauskommt, die Ermittlung der Konstanten $A$ und $B$ und damit eine weitere Kontrolle theoretischer Ansätze. Über weitere Resultate wird bei späterer Gelegenheit berichtet werden.

\section{Beobachtungen an den Raman-Linien binärer und ternärer Gemische von Aceton, Methyl- alkohol und Schwefelkohlenstoff}

Von Maria Joerges und Alexander Nikuradse Institut für Elektronen- und Ionenforschung der Techn. Hochschule München

(Z. Naturforschg. 4 a, 228 [1949]; eingeg. am 21. April 1949)

1. Die Untersuchung der Raman-Frequenzen binärer Gemische aus Methylalkohol und Aceton sowie ternärer Gemische von Methylalkohol, Aceton und Schwefelkohlenstoff zeigt an den Frequenzen der betreffenden Substanzen Verschiebungen, die eine H-Brückenbildung Aceton-Methylalkohol wahrscheinlich machen.

2. Die Untersuchung der Intensitätsverhältnisse einiger Raman-Linien der genannten Gemische lassen den Schluß zu,

a) daf das Assoziat ungefähr im Verhältnis $1: 4$ Moleküle Aceton : Methanol gebildet wird und

b) daß das bei eben diesem Konzentrationsverhältnis ein Minimum erreichende Streuvermögen von Aceton gegenüber Methanol wohl deswegen so verringert wird, weil durch die Umlagerung des AcetonMoleküls durch mehrere Methanol-Moleküle die für die Intensität maßgebende Größe $\left(\frac{\partial \alpha}{\partial q}\right)_{0}$ stärker beeinflußt wird - und zwar verringert - als die durch die Anlagerung einzeln nicht so stark beeinflußten Methanolmoleküle.

\title{
BERICHTE
}

\section{Die Supernovae des Milchstraßensystems}

$\mathrm{I}$ n der modernen astrophysikalischen Forschung der letzten zehn Jahre gibt es kaum ein Problem, das weit über die Grenzen der Astronomie hinaus eine so weitgehende Beachtung gefunden hat wie das Wesen der Supernovae ${ }^{1}$. Es handelt sich dabei um eine besondere Klasse der Neuen Sterne, deren Erscheinung sich anfangs ausschließlich auf ferne Sternsysteme zu beschränken schien, die aber neuerdings auch in der Milchstraßenwelt nachgewiesen werden konnte.

$$
\text { 1. Novae und Supernovae }
$$

Die gewöhnlichen Norae bilden in der Milchstralie und in benachbarten Welteninseln keine sonderliche
Seltenheit. Allein im Andromedanebel leuchten in jedem Jahre etwa 20 Novae auf, während im Milchstraßensystem die Zahl der jährlichen Erscheinungen mit rund 25 anzunehmen ist, wovon aber nur ein geringerProzentsatz durch die Beobachtung erfaßt wird. Insgesamt sind in den letzten 400 Jahren in der Milchstraße annähernd 100 Novae ${ }^{2}$ aufgezeichnet worden. denen in außergalaktischen Nebeln aus den letzter

1 Vgl. K. Lundmark. Supernovae. Vischr. Astro nom. Ges. 47, 243 [1939].

2 Vgl. S. Tuchenhagen, Die Neuen Sterne: Diss. Berlin 1938. 